\title{
MOLECULAR RADIO LINE OBSERVATIONS OF MASS LOSS FROM RED GIANTS
}

\author{
H. Olofsson \\ Onsala Space Observatory, S-43900 Onsala, Sweden
}

\begin{abstract}
The number of molecules detected at radio wavelengths in envelopes around red giants stands presently at 36 . Among these $\mathrm{OH}$ and $\mathrm{CO}$ have proven to be the most useful for the study of the physical characteristics of a circumstellar envelope. The mass loss rate of the central star can be relatively accurately estimated and it appears possible to trace its evolution with time. Also fascinating objects in transition from the red giant phase to the planetary nebula phase are becoming observationally accessible.
\end{abstract}

\section{INTRODUCTION}

Our knowledge of the asymptotic giant branch (AGB) and planetary nebulae (PNe) phases of late stellar evolution, as well as the transition stage, has increased substantially in recent years. The completion of new millimetre wave instruments and the data base provided by IRAS have made molecular radio lines an important, and in some cases unique, tool in this research. It is possible to follow the evolution of the "slow wind" mass loss as the star ascends the AGB, as well as its termination during the proto-PNe phase when the mass loss appears to change its character. The consequences for the chemical evolution of the Galaxy and the supernova rate are gradually becoming apparent. The circumstellar gas/dust envelopes (CSEs) formed by the mass loss are also the sites of a complex chemistry, whose effects beyond the red giant phase have yet to be established.

We will in this review concentrate on what has and can be learnt in this area through the use of $\mathrm{OH}$ and $\mathrm{CO}$ radio line observations. The large number of recent papers has made it impossible to properly reference all works, in particular the historical ones. We therefore in most cases mention only the most recent papers and presuppose that they contain proper references to previous works. A recent extensive review of radio and infrared observations of CSEs was given by Olofsson (1988).

\section{MOLECULAR RADIO LINE OBSERVATIONS}

Both the number of objects detected in circumstellar molecular emission and the number of circumstellar molecular species have increased substantially during the last few years. This has been possible due to the availability of the IRAS data base and the completion of new sensitive millimetre wave telescopes.

\subsection{Circumstellar molecules}

Table 1 summarizes the present situation for circumstellar molecules detected at radio wavelengths. The total number now amounts to 36 (two of the 
identifications are tentative). If we include those detectable only at infrared wavelengths $\left(\mathrm{H}_{2}, \mathrm{CH}_{4}, \mathrm{C}_{2} \mathrm{H}_{2}, \mathrm{C}_{2} \mathrm{H}_{4}, \mathrm{SiH}_{4}\right)$, the total number of circumstellar molecular species stands presently at 41 . It is evident that the C-rich CSEs are apparently most productive, but the situation is improving also for the O-rich CSEs. However, it should be noted that for the C-rich, as well as for the O-rich, CSEs the scene is dominated by a single object, the extreme nearby carbon star IRC+10216 and the remarkable bipolar nebula $\mathrm{OH} 231.8+4.2$, respectively.

Table 1 Molecules Detected at Radio Wavelengths in Circumstellar Envelopes

\begin{tabular}{|c|c|c|}
\hline \multirow[t]{2}{*}{ Molecule } & \multicolumn{2}{|c|}{ Number of Sources } \\
\hline & C-rich & O-rich \\
\hline $\mathrm{CO}$ & $\approx 125$ & $\approx 100$ \\
\hline $\mathrm{CN}$ & 3 & \\
\hline CS & 6 & 3 \\
\hline SiO("thermal") & 4 & $\approx 30$ \\
\hline SiS & 4 & 1 \\
\hline $\mathrm{SiC}_{2}$ & 4 & \\
\hline SO & & 7 \\
\hline $\mathrm{SO}_{2}$ & & 16 \\
\hline $\mathrm{H}_{2} \mathrm{~S}$ & & 15 \\
\hline OCS & & 1 \\
\hline $\mathrm{HCO}^{+}$ & 3 & 2 \\
\hline $\mathrm{HCN}$ & $\approx 50$ & 13 \\
\hline $\mathrm{CH}_{3} \mathrm{CN}$ & 3 & \\
\hline HNC & 5 & 2 \\
\hline $\mathrm{NH}_{3}$ & 2 & 1 \\
\hline $\mathrm{HC}_{3} \mathrm{~N}$ & 6 & \\
\hline $\mathrm{HC}_{5} \mathrm{~N}$ & 4 & \\
\hline $\mathrm{HC}_{7} \mathrm{~N}$ & 2 & \\
\hline $\mathrm{HC}_{9} \mathrm{~N}$ & 1 & \\
\hline $\mathrm{HC}_{11} \mathrm{~N}$ & 1 & \\
\hline $\mathrm{C}_{2} \mathrm{H}$ & 4 & \\
\hline $\mathrm{C}_{3} \mathrm{H}$ & 2 & \\
\hline $\mathrm{C}_{3} \mathrm{H}_{2}$ & 3 & \\
\hline $\mathrm{C}_{4} \mathrm{H}$ & 4 & \\
\hline $\mathrm{C}_{5} \mathrm{H}$ & 1 & \\
\hline $\mathrm{C}_{6} \mathrm{H}$ & 1 & \\
\hline $\mathrm{C}_{3} \mathrm{~N}$ & 5 & \\
\hline $\mathrm{C}_{2} \mathrm{~S}$ & 1 & \\
\hline $\mathrm{C}_{3} \mathrm{~S}$ & 1 & \\
\hline $\mathrm{HSiC}_{2}\left(\mathrm{HSC}_{2}\right)(?)$ & 1 & \\
\hline $\mathrm{NaCl}$ & 1 & \\
\hline $\mathrm{AlCl}$ & 1 & \\
\hline $\mathrm{KCl}$ & 1 & \\
\hline AlF (?) & 1 & \\
\hline $\mathrm{OH}$ (maser) & & $>1000$ \\
\hline $\mathrm{H}_{2} \mathrm{O}$ (maser) & 4 & $\approx 200$ \\
\hline SiO (maser) & & $\approx 140$ \\
\hline
\end{tabular}


Armed with this wealth of molecular probes it is possible to study various effects of mass loss from evolved stars. From a statistical point of view it appears that the $\mathrm{OH}$ $1612 \mathrm{MHz}[\mathrm{OH}(1612)]$ maser line and the $\mathrm{CO}$ lines are the most useful probes of the CSEs. $\mathrm{OH}$ and $\mathrm{CO}$ observations of evolved objects will therefore be discussed at some length below.

\section{$2.2 \mathrm{OH}$ and $\mathrm{CO}$ observations}

2.2.1 OH. It was the detection of $\mathrm{OH}$ maser emission from red giants in the late sixties that marked the beginning of radio astronomical research on evolved stars. The subsequent observations of $\mathrm{OH}(1612)$ maser emission from $\mathrm{OH} / \mathrm{IR}$-stars have substantially contributed to, and in many cases been crucial for, our understanding of the late stages of AGB evolution (see e.g., Herman and Habing, 1985a). Their strength and the absence of extinction at $18 \mathrm{~cm}$ make them observable throughout the Galaxy. Towards the galactic centre there are observational problems with extended absorption, but this can be avoided by using interferometers (Lindqvist et al., 1987). An OH/IR-star has also been detected in an extemal galaxy, the LMC (Wood et al., 1986). The fact that their distances can be fairly accurately estimated by combining linear size (from phase lag measurements) and angular size (interferometer observations) further strengthens their usefulness (Herman et al., 1985).

Red Giants and Supergiants. OH maser emission has now been detected towards $\mathrm{O}$-rich red giants over a large range of mass loss rates: from the low mass loss rate $\left(<10^{-7} \mathrm{M}_{\theta} \mathrm{yr}^{-1}\right)$, short-period Miras and semiregulars (Dickinson et al., 1986), through the long-period Miras and even longer-period OH/IR-stars up to the very top of the AGB $\left(>10^{-4} \mathrm{M}_{\theta} \mathrm{yr}^{-1}\right)$ and perhaps beyond that (Nguyen-Q-Rieu et al., 1979; Herman and Habing, 1985b). When the ongoing surveys at Parkes and Arecibo of new OH/IR-sources, based on colour-selected IRAS sources (Lewis et al., 1985; Sivagnanam and Le Squeren, 1986), are completed the number of red giants detected in circumstellar OH(1612) emission will be well above a thousand (te Lintel Hekkert, 1987; Eder et al., 1988). A number of wellknown supergiants are also detected in $\mathrm{OH}$ maser emission: VY CMa, NML Cyg, S Per, VX Sgr, and IRC+10420 (this may be a proto-PNe) (Cohen et al., 1987; Diamond et al., 1987).

Proto-PNe and PNe. Habing et al. (1987) proposed that the non-variable $\mathrm{OH} / \mathrm{IR}$-stars with large mass loss rates are proto-PNe, i.e., they have or will shortly cease losing mass and increasingly hotter regions of the star will become uncovered. Modelling of the infrared radiation from these objects supports this suggestion (Bedijn, 1987). However, only upper limits to radio continuum emission were obtained until recently when Pottasch et al. (1987) managed to detect 2 stars at 2 and $6 \mathrm{~cm}$. Both share, with the previously only known $\mathrm{OH}$-emitting PNe $\mathrm{Vy} 2-2$ (Davis et al., 1979), the common characteristic that only a single $\mathrm{OH}(1612)$ feature is present. Recently, Payne et al. (1988) searched 79 PNe with the meagre result of only one detection, the very young PN NGC6302. Once again only one $\mathrm{OH}$ feature was present. To this list we should add M1-92 (Lepine and Nguyen-Q-Rieu, 1974), and possibly OH5.89-0.39 (Zijlstra and Pottasch, 1988). Vy2-2 also shows the rotationally excited $\mathrm{OH}\left({ }^{2} \Pi_{3 / 2}, \mathrm{~J}=5 / 2\right) 6035 \mathrm{MHz}$ line in maser emission (Jewell et al., 1985). In NGC6302 the 6030 and $6035 \mathrm{MHz}$ lines were seen in absorption (Payne et al., 1988). It seems that the statistics of $\mathrm{OH}$-emitting (proto-) PNe can probably be improved substantially in the future by examining single-feature sources from the ongoing surveys of $\mathrm{OH} / \mathrm{IR}$-stars. There are at least three other $\mathrm{OH}$-sources that appear to be in transition from the tip of the AGB and to the left in the HR-diagram, IRC+10420 (probably a supergiant; Lewis et al., 1986), OH231.8+4.2 (Morris et al., 1982), and IRAS16342-3814 (Likkel and Morris, 1988). 
2.2.2 CO. The CO millimetre wave lines have become an increasingly important tool for the study of CSEs. They have the advantages of being fairly easily modelled, and being detectable in $\mathrm{O}$-rich as well as C-rich CSEs. Their main disadvantages are the limited observational space (see $\$ 4.2$ ), and the fact that they are easily excited and hence may mask more complicated density structures.

Red Giants and Supergiants. The number of CSEs detected in CO emission has increased dramatically during the last few years, and stands presently at well above 200 , divided roughly equally between $\mathrm{O}$-rich and $\mathrm{C}-$ rich objects. The mass loss rate range covered is $\left\langle 10^{-7} \mathrm{M}_{\theta} \mathrm{yr}^{-1}\right.$ to $>10^{-4} \mathrm{M}_{\theta} \mathrm{yr}^{-1}$ and seems to be independent of chemical composition (Knapp, 1987). Many surveys have been based on the IRAS point source catalogue, but also other criteria have been used. Pre-IRAS surveys were performed by Knapp and Morris (1985) and Wannier and Sahai (1986). Based on IRAS observations Zuckerman and Dyck (1986a,b, 1988), Zuckerman et al. (1986), Leahy et al. (1987), Likkel et al. (1987), and Nguyen-Q-Rieu et al. (1987), considerbly increased the number of detected CSEs. In particular, Nguyen-Q-Rieu et al. managed to detect fairly distant ones $(\approx 5 \mathrm{kpc})$ using the IRAM $30 \mathrm{~m}$ telescope. Recently $13 \mathrm{OH} / \mathrm{IR}$-stars were detected (Heske, priv. comm.). Many of the above mentioned surveys have concentrated on obscured objects, where little is known about the central stars. Olofsson et al. $(1987,1988$ a) approached this problem by observing bright carbon stars with relatively well defined photospheric

characteristics. CO emission from supergiants is usually quite weak, but several of them have nevertheless been detected: $\alpha$ Ori (Huggins, 1987), VY CMa (Zuckerman and Dyck, 1986a), NML Cyg (Zuckerman, priv. comm.), IRC+10420 (Bachiller et al., 1988), and AFGL2343 (Zuckerman and Dyck, 1986b).

Proto-PNe and PNe. Up to recently only a few proto-PNe were detected in CO, and searches towards true PNe were mostly negative. However, also here the situation has improved, and the following proto-PNe are now detected: CRL618, M2-9 and HD44179 (Bachiller et al., 1988), CRL2688 (Kawabe et al., 1987), M1-92 (Knapp, 1986), and $\mathrm{OH} 231.8+4.2$ (Morris et al, 1987). The following objects are also potential proto-PNe objects: IRAS09371+1212 (Forveille et al., 1987), HD161796, SAO163075, and 89 Her (Likkel et al., 1987). IRC +10420 probably also belongs to this class (Bachiller et al., 1988). The PNe detected in CO are: NGC2346 (Huggins and Healy, 1986a; Healy and Huggins, 1988), NGC6302 (Zuckerman and Dyck, 1988), NGC6720 (Huggins and Healy, 1986a), NGC7027 (Masson et al., 1985), NGC7293 (Huggins and Healy, 1986b), and IRAS21282+5050 (Likkel et al., 1988). Also here the statistics are likely to improve considerably when new sensitive millimetre wave observations, guided by IRAS data, are performed.

\section{CHARACTERISTICS OF THE CIRCUMSTELLAR ENVELOPES}

We will in this section try to summarize our current knowledge of the characteristics of CSEs, e.g., the geometry, the kinematics, the density structure, the kinetic temperature, the lifetime, etc. The main emphasis is put on what has been learnt by using molecular radio line data.

\subsection{The geometry}

It has been known for some time that when observed with sufficient spatial resolution (using IR speckle interferometry or direct IR imaging) or with polarimetry the inner regions of many CSEs have a clearly non-spherical geometry (Dyck, 1987; Ridgway and Keady, 1988). In the few cases where the central region becomes visible in the optical, the structure is often bipolar, e.g., CRL618 (Schmidt and Cohen, 1981), CRL2688 (Ney et al., 1975), OH231.8+4.2 (Reipurth, 1987), 
IRAS09371+1212 (Hodapp et al., 1988, Rouan et al., 1988), and M2-9 (Aspin et al., 1988). It has also been known that the presumed end-products of AGB evolution, PNe, have mainly non-spherical geometries (see e.g., Zuckerman and Aller, 1986; Balick, 1987). Zuckerman and Aller (1986) carefully examined the morphology of $108 \mathrm{PNe}$ and concluded that $\approx 50 \%$ have a bipolar structure and $\approx 30 \%$ have an elliptical symmetry.

When it comes to the structure of the intermediate stage, the CSEs, the situation is much more unclear and hence unsatisfying. The distribution of $\mathrm{H}_{2} \mathrm{O}$ masers associated with Mira variables are in many cases non-spherical (Lane et al., 1987). This is also the case for the $\mathrm{OH}$ main line and $\mathrm{H}_{2} \mathrm{O}$ masers surrounding the supergiant VX Sgr (Chapman and Cohen, 1986). A number of OH/IR-stars $(\approx 30)$ have been mapped in the OH(1612) line (Bowers et al., 1983; Diamond et al., 1985; Herman et al., 1985, Welty et al., 1987). It appears that the $\mathrm{OH}-$ emitting region is circularly symmetric (a thin shell, $\leq 300 \mathrm{AU}$, at a radius of $\approx 1500 \mathrm{AU}$ ) and by inference spherically symmetric, but there is considerable clumpiness. This symmetry agrees with the infrared speckle interferometry and imaging data on several $\mathrm{OH} / \mathrm{IR}$ - stars obtained by Cobb and Fix (1987).

Single dish observations of thermal molecular line emission are usually not able to resolve any structure in the CSEs. Furthermore, millimetre wave interferometer data are sparse due to the limited number of instruments and the observational difficulties, and hence our knowledge is based on very scattered data. The CSE of the carbon star IRC +10216 has been mapped on several occasions. It appears spherical in the $\mathrm{CO}(\mathrm{J}=1-0,2-1)$ lines out to $\approx 40000 \mathrm{AU}$ (Huggins et al., 1988), as well as in the $\mathrm{HCN}(\mathrm{J}=1-0)$ line out to $\approx 5000 \mathrm{AU}$ (Bieging et al., 1984). On the contrary, $\mathrm{CO}(\mathrm{J}=1-0)$ maps of the carbon star $\mathrm{V}$ Hya show clear evidences for a bipolar outflow, as well as an extended symmetric CSE (Kahane et al., 1988b; Tsuji et al., 1988). The near-infrared spectrum provides evidence for high-velocity motions, $\approx 100 \mathrm{~km} \mathrm{~s}^{-1}$ (uncorrected for inclination) (Sahai and Wannier, 1988).

Observations of CSEs around objects believed to be in the transition phase often reveal complicated structures. The bipolar nebula $\mathrm{OH} 231.8+4.2$ appears to have a CSE similar in shape to that of V Hya. A CO $(\mathrm{J}=1-0)$ map reveals a bipolar outflow, aligned with the optical image, with an outflow velocity exceeding $140 \mathrm{~km} \mathrm{~s}^{-1}$ (Morris et al., 1987). The OH maser maps are extended along the same axis (Morris et al., 1982). However, the dominant CO emission, as well as that of most other molecules, originates from a core that is unresolved at a resolution of 21" (Morris et al., 1987). For a similar object, IRAS16342-3814, Likkel and Morris (1988) conclude from $\mathrm{OH}$ and $\mathrm{H}_{2} \mathrm{O}$ observations that it is an evolved star with a bipolar outflow. The outflow velocity is about $130 \mathrm{~km} \mathrm{~s}^{-1} \cdot \mathrm{CO}(\mathrm{J}=1-0)$ observations of another bipolar nebula, CRL2688, reveal expanding lobes around the optical nebulosity, as well as a large, cold CSE that is circularly symmetric (Heiligman et al., 1986; Kawabe et al., 1987). Nguyen-Q-Rieu et al. (1986) and Beiging and Nguyen-Q-Rieu (1988b) have found evidence for a disk- or toroidal-like density distribution lying perpendicular to the optical lobes from $\mathrm{NH}_{3}(1,1)$ and $\mathrm{HCN}(\mathrm{J}=1-0)$ observations, respectively. The CSE of CRL618, also a bipolar nebula, appears circularly symmetric in the $\operatorname{CO}(\mathrm{J}=1-0,2-1)$ lines, but the kinematics suggest that the $\mathrm{CO}$ gas takes part in a bipolar outflow (Bachiller et al., 1988). Finally, Masson et al. (1985) have mapped the $\mathrm{CO}(\mathrm{J}=1-0)$ emission associated with the PN NGC7027. They conclude that the inner envelope is ellipsoidal in shape, and the ionized gas is expanding faster along the low density polar axis. However, at velocities close to the stellar velocity only 5-10\% of the total flux appear in the interferometer map, suggesting an extended, resolved CSE. In the somewhat more developed PN 
NGC2346, Bachiller et al. (1988) found remnant CO gas that participates in the bipolar motion seen in the visible. In NGC7293 the neutral gas is associated with the outer regions of the ring-like optical nebulosity, and it appears to be assembled in two expanding toroids (Huggins and Healy, 1986b).

\subsection{The kinematics}

The kinematics of a pulsating red giant atmosphere are extremely complicated with both rising and falling matter giving rise to shock waves (Hinkle et al., 1982). This is apparently also the case in the region between the pulsating atmosphere and the expanding CSE as inferred from SiO maser observations (Nyman and Olofsson, 1986). In fact, also the inner part of the CSE is expected to have a fairly complex velocity distribution, due to episodic mass ejection and acceleration (Keady et al., 1988).

On the other hand, the extended part of a CSE is expected to simply expand with a constant velocity since here no acceleration agents are effective. It now appears that the mechanism responsible for, at least, the final characteristics of the mass loss is radiation pressure on grains (the gas is dragged along) formed in the extended atmospheres of the stars (see Jura, these proceedings), i.e., the gas gains momentum from the grains. In the extemal parts of a CSE the grains drift supersonically through the gas with a velocity, $v_{d}$, obtained by equating the radiative force and the drag force (Kwok, 1975),

$$
\mathrm{v}_{\mathrm{d}}=\left(\frac{\mathrm{v}_{\mathrm{e}} \mathrm{QL}}{\mathrm{clM}}\right)^{0.5}
$$

where $v_{e}$ is the gas expansion velocity, $Q$ is the ratio of the radiative momentum transfer cross section to the geometric cross section of the grains (averaged over frequency), $L$ is the luminosity of the star, $c$ is the speed of light, and $M$ is the mass loss rate. Papoular and Pegourie (1986) have estimated that $v_{d}$ can be as high as 20 $\mathrm{km} \mathrm{s}^{-1}$, but for high mass loss rate CSEs it is expected to be at most a few $\mathrm{km} \mathrm{s}^{-1}$. In the case of a negligible drift velocity the final expansion velocity of the gas is given by (Jura, 1984),

$$
v_{e}=\left(\frac{x_{d} L}{2 \pi r_{c} c}\right)^{0.5}
$$

where $X_{d}$ is the dust opacity per unit mass (in fact, $\|_{d} L$ is weighted over all frequencies), and $r_{c}$ is the dust condensation radius. It is expected from this that $v_{e}$ should be only mildly dependent on the stellar luminosity since $r_{c}$ is roughly proportional to $\mathrm{L}^{0.5}$.

Most molecular line observations are consistent with a well defined terminal velocity for the gas that falls within a limited range. The compilation of Knapp (1987) shows that for 136 stars detected in CO emission, $90 \%$ lie within $5 \leq \mathrm{v}_{\mathrm{e}} \leq 25 \mathrm{~km} \mathrm{~s}^{-1}$ and $\left\langle v_{e}\right\rangle \approx 17$ and $18 \mathrm{~km} \mathrm{~s}^{-1}$ for $\mathrm{O}-$ and $\mathrm{C}-$ stars, respectively. The terminal velocities for $\mathrm{OH} / \mathrm{IR}-$ stars fall in the same region (Eder et al., 1988), and are only mildly dependent on the luminosity (Jura, 1984). For CO- as well as OH/IR-stars the "large terminal velocity"-sources are concentrated to the galactic plane, and they are therefore presumably younger and hence more massive (Knapp, 1987; Eder et al., 1988; Zuckerman and Dyck, 1988). In only one case, VX Sgr, the SiO, $\mathrm{H}_{2} \mathrm{O}$, and $\mathrm{OH}$ interferometer maps can be interpreted in terms of an envelope that is still accelerating at $\approx 1000 \mathrm{AU}$ (this corresponds to $\approx 50$ stellar radii), possibly due to grain growth (Chapman and Cohen, 1986). 
In the case of the transition objects it is clear from the discussion in $\$ 3.1$ that the velocity structure once again becomes very complex, e.g., due to the onset of a fast stellar wind, expanding ionization fronts that are either ionization- or density-bounded, etc. In CRL2688 there is weak high-velocity CO emission $\left(\approx 40 \mathrm{~km} \mathrm{~s}^{-1}\right)$ from a small region near the centre of the optical image (Heiligman et al., 1986; Kawabe et al., 1987). In NGC7027 the spatial resolution is high enough to conclude that similar emission originates in a layer of neutral gas shocked by the expanding HII-region (Masson et al., 1985). The presence of bipolar structures has been inferred in a few cases: V Hya, OH231.8+4.2, IRAS16342-3814, CRL618, and NGC2346 (see \$3.1). For the three former sources the outflow velocities exceed $100 \mathrm{~km} \mathrm{~s}^{-1}$. The reason for the onset of such high-velocity flow is not known. The "normal state" mass loss mechanism, radiation pressure on grains, can be excluded in this case.

It should be mentioned that the kinematics may have an appreciable effect on the excitation of molecules. A velocity gradient allows infrared photons from the hot core to leak out into the envelope and enhance the excitation through infrared pumping. This may, in the external tenuous parts, have a considerable effect also on otherwise collisionally excited molecules like $\mathrm{CO}$. In the same way it may affect the cooling of the gas (see \$3.4).

3.3 The density structure and the total mass

The density at a radius $r$ and in the direction $(\theta, \varphi)$ is intimately connected to the retarded mass loss rate, and the kinematics,

$$
\dot{M}\left(r-\int_{r_{i}}^{r} \frac{d r}{v(r, \theta, \varphi)}, \theta, \varphi\right)=4 \pi r^{2} \rho(r, \theta, \varphi) v(r, \theta, \varphi),
$$

where

$$
\rho=\mu \mathrm{m}(\mathrm{H}) \mathrm{n}\left(\mathrm{H}_{2}\right)=(1+4 \mathrm{n}(\mathrm{He}) / \mathrm{n}(\mathrm{H}))\left(2 \mathrm{n}\left(\mathrm{H}_{2}\right)+\mathrm{n}(\mathrm{HI})\right) \mathrm{m}(\mathrm{H})
$$

It is therefore in principle possible to determine the spatial and temporal characteristics of the mass loss mechanism from the density structure and the kinematics. The discussions in $\S: s 3.1$ and 3.2 indicate that these can be quite complicated. In the simplest case of spherical geometry, constant mass loss rate and expansion velocity, and $n\left(\mathrm{H}_{2}\right)>>n(\mathrm{HI})$ we obtain the following simple relation

$$
\mathrm{n}\left(\mathrm{H}_{2}\right) \approx 10^{6}\left(\frac{\ddot{M}}{10^{-6} \mathrm{M}_{\theta} \mathrm{yr}^{-1}}\right)\left(\frac{15 \mathrm{~km} \mathrm{~s}^{-1}}{\mathrm{v}_{\mathrm{e}}}\right)\left(\frac{10^{15} \mathrm{~cm}^{2}}{\mathrm{r}}\right)^{2} \mathrm{~cm}^{-3} \text {. }
$$

The evidences for a departure from the $r^{-2}$ density law are sparse. Keady et al. (1988) conclude from a detailed study of infrared CO absorption lines towards IRC+10216 that acceleration occurs in steps within 14 stellar radii and for a constant mass loss rate this means an initial density gradient steeper than $\mathrm{r}^{2}$. Also, Bedijn (1987) in his IR continuum models requires steep initial density gradients for the low mass loss rate objects in order to fit the observed spectra. Grain growth and hence acceleration could be an explanation. A mass loss rate increasing with time would also lead to a steeper density gradient. According to Bedijn this will produce observable consequences in the IR spectra only for the highest mass loss rates, $>2 \times 10^{-4} \mathrm{M}_{\theta} \mathrm{yr}^{-1}$, but this conclusion depends on the adopted $\dot{M}(t)$. Modelling of molecular line emission from the CSE of IRC+10216 requires a decreasing mass loss rate and hence a flatter density distribution (Sahai, 1987). Olofsson et al. (1988a) interpreted 
$\mathrm{CO}(\mathrm{J}=1-0)$ observations of the carbon star S Sct in terms of a detached CSE formed by a mass loss that decreased considerably $\approx 5000$ yr ago. Another carbon star, U Ant, exhibits a remarkable $\mathrm{CO}(\mathrm{J}=2-1)$ line profile that may be interpreted in the same way, but here the mass loss may have recommenced (Olofsson et al., 1988c).

An interesting question is whether the mass loss is continuous or occurs in the form of blobs of released material. A patchy density structure in the region where the acceleration starts is not unreasonable (see e.g., Bloemhof et al., 1985). Thermal instabilities may play a role here (Stencel et al., 1986; Muchmore et al., 1987). Alcock and Ross $(1986 \mathrm{a}, \mathrm{b})$ have addressed this problem by examining the maps and spectra of $\mathrm{SiO}$ and $\mathrm{OH}(1612)$ masers. They suggest that the mass loss occurs in blobs of high density material that in the region of the $\mathrm{OH}(1612)$ masers have somehow developed into pancakes. High spectral resolution observations of $\mathrm{OH}(1612)$ masers are not inconsistent with this model (Cohen et al., 1987; Fix, 1987).

The total mass of a CSE within a radius $R_{e}$ is approximately given by,

$$
M\left(R_{e}\right) \approx \frac{\dot{M} R_{e}}{v_{e}}=2 \times 10^{-3}\left(\frac{\dot{M}}{10^{-6} M_{\theta} \mathrm{yr}^{-1}}\right)\left(\frac{R_{e}}{10^{17} \mathrm{~cm}^{-}}\right)\left(\frac{15 \mathrm{~km} \mathrm{~s}^{-1}}{\mathrm{v}_{\mathrm{e}}}\right) \mathrm{M}_{\theta}
$$

Thus, even a huge envelope like that of IRC+10216, where $\dot{M} \approx 2 \times 10^{-5} \mathrm{M}_{\Theta} \mathrm{yr}-1$, and $v_{e} \approx 15 \mathrm{~km} \mathrm{~s}^{-1}$, has a mass of only $0.5 \mathrm{M}_{\theta}$ within $R_{e}=10^{18} \mathrm{~cm} . M\left(R_{e}=" \infty "\right)$ is of course an important parameter since it provides us with the total amount of mass lost during the AGB phase so far. However, to observationally directly estimate this quantity is not so simple. Adopting a certain probe will only yield the mass within a limited region, determined by chemical and excitation processes, unless uncertain extrapolations are being made. For most molecules the fractional abundance, $f(X, r)=n(X, r) / n\left(H_{2}, r\right)$, has a radial variation that is sufficiently complex to make them useless in this connection (Bieging and Nguyen-Q-Rieu, 1988a). In the case of the most stable species, CO, the extent of the detectable CSE is determined by photodissociation. Mamon et al. (1988) have carefully examined this problem by including self,$- \mathrm{H}_{2}-$, and dust shielding. The extent of the $\mathrm{CO}$ envelope (defined as $f\left(C O, R_{e}\right)=f(C O) / 2$, where $f(C O)$ is the freeze-out photospheric abundance) is a complicated function of $\dot{M}$ and $v_{e}$. An approximate relation for $\dot{M}>10^{-7} \mathrm{M}_{\theta} \mathrm{yr}^{-1}$, $v_{e}=15 \mathrm{~km} \mathrm{~s}^{-1}$, and $\mathrm{f}(\mathrm{CO})=4 \times 10^{-4}$, is given by,

$$
\mathrm{R}_{\mathrm{e}}(\mathrm{CO}) \approx 5 \times 10^{16}\left(\frac{\dot{\mathrm{M}}}{10^{-6} \mathrm{M}_{\theta} \mathrm{yr}^{-1}}\right)^{0.6} \mathrm{~cm} \text {. }
$$

This gives considerably lower values for the size of the $\mathrm{CO}$ envelope than does the model of Morris and Jura (1983). It is also consistent with modelling of circumstellar $\mathrm{CO}$ emission (Sopka et al., 1988). The dependence of $\mathrm{R}_{e}(\mathrm{CO})$ on $\mathrm{f}(\mathrm{CO})$ and $v_{e}$ is approximately given by $\left(\mathrm{f}(\mathrm{CO}) / v_{e}\right)^{0.5}$. The corresponding time scale is given by,

$$
t(C O) \approx \frac{R_{e}(C O)}{v_{e}} \approx 10^{3}\left(\frac{\dot{M}}{10^{-6} M_{\theta} \mathrm{yr}^{-1}}\right)^{0.6} \mathrm{yr} \text {, }
$$

for $\mathrm{v}_{\mathrm{e}}=15 \mathrm{~km} \mathrm{~s}^{-1}$. Thus, we can only estimate the mass lost during the last $\approx 300$ years for $\dot{M}=10^{-7} \mathrm{M}_{\theta} \mathrm{yr}^{-1}$ and $\approx 13000$ years for $\dot{\mathrm{M}}=5 \times 10^{-5} \mathrm{M}_{\theta} \mathrm{yr}^{-1}$. The total detectable mass would be,

$$
\mathrm{M}(\mathrm{CO}) \approx \dot{\mathrm{M}} \mathrm{t}(\mathrm{CO}) \approx 1.2 \times 10^{-3}\left(\frac{\dot{\mathrm{M}}}{10^{-6} \mathrm{M}_{\theta} \mathrm{yr}^{-1}}\right)^{1.6} \mathrm{M}_{\theta}
$$


This will exceed $1 \mathrm{M}_{\theta}$ only for $\dot{\mathrm{M}}>7 \times 10^{-5} \mathrm{M}_{\theta} \mathrm{yr}^{-1} . \dot{\mathrm{M}}$ derived from $\mathrm{CO}$ millimetre wave data is for an unresolved envelope dependent on the distance, $D$, squared (see $\$ 4.2$ ), and hence $M(C O)$ is roughly dependent on $D^{3}$ and thus very uncertain.

Observations of material outside $\mathrm{R}_{\mathrm{e}}(\mathrm{CO})$ can in principle be performed in the HI 21 $\mathrm{cm}$ line because sufficiently far out $\mathrm{H}_{2}$ is also photodissociated (Glassgold and Huggins, 1983). However, these observations are not easily performed (see Knapp, 1985). Only recently were the first detections of $\mathrm{HI}$ in CSEs made. Bowers and Knapp $(1987,1988)$ detected HI in emission towards $\alpha$ Ori and omicron Ceti. In the case of $\alpha$ Ori the CSE is not representative since the star is so warm that hydrogen exists essentially in atomic form throughout the envelope (Glassgold and Huggins, 1983). omicron Ceti is cooler but still about $30 \%$ of the hydrogen is in atomic form when it leaves the star. Towards other CSEs only upper limits have been obtained (Knapp, 1985; Schneider et al., 1987). In the case of IRC+10216 Bowers and Knapp (1987) conclude that the CSE cannot be much larger than the size measured in CO. Detections of HI in PNe have been made: NGC6302 (Rodriguez et al., 1985), NGC6790 (Gathier et al., 1986), IC418 (Taylor and Pottasch, 1987), and IC4997 (Altschuler et al., 1986). Another potentially useful probe (and perhaps the best) for the total mass of a CSE is provided by submillimetre observations of dust emission (Sopka et al., 1985).

\subsection{The temperature structure}

The temperature of the grains is determined by the balance between the absorbed stellar radiation energy at "short" wavelengths and the reradiated energy at longer wavelengths. In the optically thin limit this results in a radial dependence for the grain temperature given by,

$$
T_{d}(r) \alpha r^{-2 /(4+p)},
$$

where $p$ describes the wavelength dependence of the grain emissivity in terms of $\lambda^{-p}$ (Sopka et al., 1985). For C-rich CSEs it appears that $\mathrm{p} \approx 1$ over a wide wavelength range (Jura, 1986; Zuckerman and Dyck, 1986b), while for O-rich CSEs the dependence on wavelength is more complicated (Zuckerman and Dyck, 1986b). Close to the star the temperature may exceed that obtained from the optically thin approximation. Nevertheless eq.(10) combined with $\mathrm{T}_{\mathrm{d}} \approx 1000 \mathrm{~K}$ at the dust condensation radius (Ridgway et al., 1986; Bedijn, 1987; Keady et al., 1988) should give a reasonable description of the radial dependence of the dust temperature.

The gas kinetic temperature is independent of the dust temperature. The heating of the gas is due to the supersonic streaming of the dust particles through the gas, i.e., gas-grain collisions (Goldreich and Scoville, 1976),

$$
\frac{d q_{d u s t}}{d t}=\frac{1}{2^{n}} \sigma_{d} \rho v_{d}^{3}
$$

where $n_{d}$ is the dust particle density, $\sigma_{d}$ is the geometrical cross section of the grains, and $\rho$ is the gas density. It is dependent on the mass loss rate (see eq.(1)) and may therefore vary considerably from star to star. The cooling is due to adiabatic expansion and radiative cooling via molecular rotational lines (mainly $\mathrm{CO}$ and $\mathrm{H}_{2} \mathrm{O}$ in $\mathrm{C}$-rich and $\mathrm{O}$-rich CSEs, respectively; Goldreich and Scoville, 1976; Kwan and Linke, 1982), which in its turn is dependent on the dynamics through the escape of photons (Tielens, 1983). It therefore appears that the radial dependence of the kinetic temperature has to be obtained by solving the energy balance equation, 


$$
\frac{\mathrm{dT}_{\mathrm{k}}}{\mathrm{dr}}=\frac{\gamma-1}{\mathrm{knv}} \frac{\mathrm{dq}}{\mathrm{dt}} \mathrm{dust}-2(\gamma-1) \frac{\mathrm{T}_{\mathrm{k}}}{\mathrm{r}}-\frac{\gamma-1}{\mathrm{knv}} \frac{\mathrm{dq}_{\mathrm{rad}}}{\mathrm{dt}}
$$

for each individual CSE ( $\gamma$ is the ratio of specific heats, $k$ is the Boltzmann constant, $\mathrm{n}$ is the gas particle density, and $\mathrm{v}$ is the gas expansion velocity). Huggins et al. (1988) recently did this for IRC +10216 by using $C O(J=1-0,2-1)$ data as a constraint. A reasonable fit to their result is given by (assumed distance $200 \mathrm{pc}$ ),

$$
\mathrm{T}_{\mathrm{k}}(\mathrm{r}) \approx 60\left(\frac{\mathrm{r}}{10^{16} \mathrm{~cm}}\right)^{-0.7 \mathrm{~K}}
$$

with a sligthly weaker dependence on $r$ for $r>2 \times 10^{17} \mathrm{~cm}$. For the lower mass loss rate CSEs, with high drift velocities (see eq.(1)), we would expect $T_{k}$ to be much higher than this, at least in the inner regions. In fact, it seems that eq.(13) has to be modified upwards also in the inner parts of the IRC+10216 envelope by as much as a factor of three (Sahai and Wannier, 1985; Sahai, 1987). On the other hand, the high mass loss rate CSEs could be significantly cooler. The presence of ice features at $3.1 \mu \mathrm{m}$ in five CSEs are consistent with this (Rouan et al., 1988; van der Veen et al., 1988). Nevertheless eq.(13) has been widely used as a standard for CSEs of arbitrary mass loss rate and chemical composition. A second heating process may come into play in the external parts of a CSE, photoelectric heating due to the interstellar radiation field. Huggins et al. (1988) included this in the energy balance equation in an apparently successful attempt to explain the large size of the $\mathrm{CO}$ emitting region around IRC+10216. This may be an important process also for (proto-) PNe.

\subsection{The post-AGB lifetime}

One of the most fascinating stages of late stellar evolution is the transition from the tip of the AGB to the PN phase. Here we encounter the termination of the mass loss, the uncoverage of the bright stellar core, and the interaction between the ionized wind and the gradually dispersing CSE remnant of the red giant phase. However, molecular line observations of this phase give a somewhat confused picture. Non-variable $\mathrm{OH} / \mathrm{IR}$-stars are likely $\mathrm{PNe}$-progenitors, but only two have been detected in radio continuum so far (see \$2.2.1). The same number of $\mathrm{OH}$-emitting $\mathrm{PNe}$ are known. $\mathrm{CO}$ emission appears to be a comparatively better diagnostic, but also here the results are contradictory. Some sources are strong and also rich in other molecular species, but most of them are exceedingly weak or undetectable. A case in point is CRL 618 where Bujarrabal et al. (1988) have found a chemistry very different from those of CRL2688 and IRC+10216. It appears that the molecular characteristics of these transition objects must be very sensitively dependent on the details of the evolution.

No doubt, part of the observational problem stems from the short time scales involved. The optical depth of a CSE, and hence the protection of the molecules, decreases rapidly after the termination of the mass loss as the following calculation shows. The column density of molecular hydrogen $N\left(H_{2}\right)=n\left(H_{2}, R_{i}\right) R_{i}$ where $R F v_{e} t$ is the inner radius of the CSE and $t$ is the time since the mass loss ceased, is assumed to be related to the extinction via the relation $\mathrm{N}\left(\mathrm{H}_{2}\right)=0.94 \times 10^{21} \mathrm{~A}_{\mathrm{v}}$ $\mathrm{cm}^{-2} \mathrm{mag}^{-1}$ which seems applicable to interstellar clouds (Bohlin et al., 1978). Combined with the mass loss rate ,eq.(3), this gives,

$$
\mathrm{A}_{\mathrm{v}} \approx 20\left(\frac{\dot{M}}{10^{-\sigma} \mathrm{M}_{\theta} \mathrm{yr}^{-1}}\right)\left(\frac{\mathrm{yr}}{\mathrm{t}}\right) \mathrm{mag} \text {. }
$$

That is, for low mass loss rates the dispersion time is very short and even for 
$\dot{M}=10^{-4} \mathrm{M}_{\theta} \mathrm{yr}^{-1}$ it takes only $\approx 2000$ years to reach $\mathrm{A}_{\mathrm{v}}=1$. The increasing penetration of the UV flux, both from the inside and from the outside, is bound to have profound effects on the chemistry. Sun and Kwok (1987) discussed the $\mathrm{OH}$-emitting properties of a CSE during the transition stage.

\subsection{Elemental abundances and isotope ratios}

Elemental abundances and isotope ratios are two important time keepers of stellar evolution. However, studies of molecular line emission from the surrounding envelopes have made only marginal contributions to this area. In particular, elemental abundances are difficult to determine in this way with any confidence. For instance in the case of the molecular factories IRC+10216, CRL618, and CRL.2688 it is in principle only possible to say that the objects are C-rich. There are clear differences in the molecular abundances between the sources but this can probably be attributed to different environmental conditions for the CSEs rather than differing elemental abundances of the central stars (Bujarrabal et al., 1988). That is, qualitative conclusions are in some cases possible, but never quantitative ones. This is because we have very little knowledge about how photospheric abundances are filtered into circumstellar abundances through a highly uncertain envelope chemistry involving, for instance, grains and photoinduced processes (Omont, 1987).

A case in point is the $[\mathrm{HCN}] /[\mathrm{CO} \mid$ abundance ratio which, for an equilibrium stellar atmosphere chemistry, should be proportional to the carbon-excess, i.e., $[\mathrm{C}|/| \mathrm{O}]-1$, and hence could be used as a measure of this quantity for $\mathrm{C}$-rich objects (from this point of view one would not expect $\mathrm{HCN}$ in $\mathrm{O}$-rich objects). Olofsson et al. (1988b) have tested this by observing both species towards 22 stars with reasonably well determined photospheric $[\mathrm{C}] /[\mathrm{O}]$-ratios. The expected general trend is born out but there is also a substantial scatter. However, the detection of $\mathrm{HCN}$ also in $\mathrm{O}$-rich objects has put some doubt on the usefulness of $\mathrm{HCN}$ as an indicator of $\mathrm{C}$-richness. Hitherto $13 \mathrm{O}$-rich stars have been detected in $\mathrm{HCN}$, several of them are normal Miras (Nercessian et al., 1988; Lindqvist et al., 1988). Lindqvist et al. even managed to detect CS in two cases, SiS in one case, and possibly HNC in one case. All three molecules are expected only in a $\mathrm{C}$-rich environment. It appears that in an $\mathrm{O}$-rich medium these molecular species are produced in a photoinduced circumstellar chemistry (Nercessian et al., 1988; Nejad and Millar, 1988).

Cases where the molecular line emission has aided in the classification of the objects are those where $\mathrm{OH}, \mathrm{H}_{2} \mathrm{O}$, and/or $\mathrm{SiO}$ masers have been detected. In general it seems safe to conclude that if any of these masers are observed then the CSE is definitely $\mathrm{O}$-rich. For instance, the recent detections of $\mathrm{H}_{2} \mathrm{O}$ maser emission towards 4 carbon stars with silicate feature CSEs leave no doubt that the surrounding CSEs are O-rich (Benson and Little-Marenin, 1987; Nakada et al., 1987, 1988; Deguchi et al., 1988).

Isotope ratios are in general possible to estimate with higher accuracy. The circumstellar ${ }^{12} \mathrm{C} / 13 \mathrm{C}$-ratios fall in the ranges expected from stellar evolution, i.e., the ratio is on the average higher for C-rich CSEs than for O-rich CSEs (Knapp and Chang, 1985; Wannier and Sahai, 1987). The ratio, $\approx 40$, obtained for IRC+10216 by Kahane et al. (1988a) using a number of optically thin lines seems secure. Jura et al. (1988) detected three ${ }^{13} \mathrm{C}$-rich carbon stars $\left({ }^{12} \mathrm{C} /{ }^{13} \mathrm{C} \approx 4\right)$ in circumstellar ${ }^{13} \mathrm{CO}(\mathrm{J}=1-0)$ emission. If anything, the circumstellar ${ }^{12} \mathrm{C} /{ }^{13} \mathrm{C}$-ratio is lower than that of the photosphere, and the ratio has been stable for at least 1000 years. It should be emphasized that ${ }^{12} \mathrm{C} /{ }^{13} \mathrm{C}$-ratios estimated from ${ }^{12} \mathrm{CO}$ and ${ }^{13} \mathrm{CO}$ millimetre wave observations are very uncertain since the excitation mechanisms and the spatial extents (if determined by self-shielding) are different. The ${ }^{18} \mathrm{O} / 17 \mathrm{O}$-ratio is significantly lower than the terrestrial value in three C-rich CSEs (Wannier and 
Sahai, 1987). The ${ }^{29} \mathrm{Si} / 28 \mathrm{Si}-,{ }^{30} \mathrm{Si} / 28 \mathrm{Si}-,{ }^{33} \mathrm{~S} /{ }^{32} \mathrm{~S}-,{ }^{34} \mathrm{~S} /{ }^{32} \mathrm{~S}-$, and ${ }^{37} \mathrm{Cl} / 35 \mathrm{Cl}-$ ratio towards IRC +10216 are all consistent with those in the solar system (Cemicharo and Guelin, 1987; Kahane et al., 1988a). A number of C-rich CSEs has been observed in the $\mathrm{HC}^{15} \mathrm{~N}(\mathrm{~J}=1-0)$ line (Andersson et al., 1987; Kahane et al., 1988a). In all cases $15 \mathrm{~N}$ is significantly underabundant with respect to the terrestrial value.

\section{ESTIMATES OF THE MASS LOSS RATE}

The mass loss rate (and its evolution with time) is the most important observational parameter for stellar evolution theories. Various methods, depending on the wavelength regime of the observational input, have been designed to estimate it (Goldberg, 1986). Often order-of-magnitude differences exist between different methods, showing the inherent difficulties involved. We will now discuss in some detail mass loss rate estimates based on $\mathrm{OH}$ and $\mathrm{CO}$ radio line observations.

\subsection{OH $1612 \mathrm{MHz}$ masers}

The $\mathrm{OH}$ abundance at the inner boundary of the CSE is negligible, and the masing $\mathrm{OH}$ molecules are believed to be formed by photodissociation of $\mathrm{H}_{2} \mathrm{O}$ molecules further out in the envelope (Huggins and Glassgold, 1982). When worked out in detail, taking into account the pumping of the masers by $35 \mu \mathrm{m}$ photons (Elitzur et al., 1976), the OH(1612) masers are expected to lie in a thin shell (at a radius determined by the mass loss rate) surrounding the central star (Netzer and Knapp, 1987; Sun and Kwok, 1987). Observationally it is found that the shell tickness is $\approx 20 \%$ of the shell radius, which in the sample of $\mathrm{OH} / \mathrm{IR}$-stars observed by Herman et al. (1985) averages at $\approx 8 \times 10^{16} \mathrm{~cm}$.

Bowers et al. (1983) obtained an important empirical relation between $\dot{M}$ and $R(O H)$, the radius of the $\mathrm{OH}(1612)$ emitting region,

$$
\dot{\mathrm{M}}=10^{-6}\left(\frac{\mathrm{R}(\mathrm{OH})}{10^{16} \mathrm{~cm}}\right)^{2} \mathrm{M}_{\Theta} \mathrm{yr}^{-1} \text {. }
$$

This gives a distance independent measure of the mass loss rate in the case of phase-lag determined sizes. However, the absolute scale rests on estimates of $\dot{M}$ obtained using various other methods. Furthermore, $\mathrm{R}(\mathrm{OH})$ determined through phase-lag measurements will only be available for a very limited number of sources (say $<100$ ) due to the extremely demanding observations.

Baud and Habing (1983) derived a relation between $\dot{M}$ and $L(O H)$, the luminosity of the $\mathrm{OH}(1612)$ maser $\left(=\mathrm{SD}^{2}\right.$, where $S$ is the flux), based on an empirical relation between $\mathrm{L}(\mathrm{OH})$ and $\mathrm{R}(\mathrm{OH})$, the assumption of a saturated maser with a given column density, and an adopted $\mathrm{OH}$ abundance,

$$
\dot{M}=4 \times 10^{-14} \frac{S^{0.5} D_{e}}{f(O H)} M_{\oplus} y^{-1} \text {, }
$$

where $S$ is given in $J y, D$ in pc, and $v_{e}$ in $\mathbf{k m ~ s}^{-1}$. This estimate is independent of other methods for estimating the mass loss rate, but it has the disadvantage of being dependent on $\mathrm{D}$ plus a number of assumptions.

Netzer and Knapp (1987) have produced a chemical model for circumstellar $\mathrm{OH}(1612)$ masers that gives the following relation between $\dot{M}, \mathbf{R}(\mathrm{OH})$, and $v_{e}$, 


$$
\dot{\mathrm{M}}=5 \times 10^{-6}\left(\frac{\mathrm{R}(\mathrm{OH})}{10^{16} \mathrm{~cm}}\right)^{1.4}\left(\frac{\mathrm{v}_{\mathrm{e}}}{15 \mathrm{~km} \mathrm{~s}^{-1}}\right)^{0.6} \mathrm{M}_{\theta} \mathrm{yr}^{-1},
$$

provided that $\mathrm{R}(\mathrm{OH})$ coincides with the radius of the $\mathrm{OH}$ number density peak. The result is in reasonable agreement with the empirical relation obtained by Bowers et al. (1983), at least for the higher mass loss rates which is also the range on which eq.(15) is based. Relation (17) implies a mass loss rate close to $10^{-4} \mathrm{M}_{\theta} \mathrm{yr}^{-1}$ for the average shell radius, $8 \times 10^{16} \mathrm{~cm}$, of $\mathrm{OH} / \mathrm{IR}$-stars obtained by Herman et al. (1985). Once again this is a distance independent estimate if phase-lag sizes are used, but it becomes proportional to D1.4 if angular sizes are used. Eq.(17) may also be used to estimate the retarded time at which the OH(1612) maser samples the mass loss rate,

$$
t(\mathrm{OH}) \approx \frac{\mathrm{R}(\mathrm{OH})}{\mathrm{v}_{\mathrm{e}}} \approx 10^{2}\left(\frac{\dot{\mathrm{M}}}{10^{-6} \dot{\mathrm{M}}_{\theta \mathrm{y}-1}^{-1}}\right)^{0.7} \mathrm{yr},
$$

for $v_{\mathrm{e}} \approx 15 \mathrm{~km} \mathrm{~s}^{-1}$. Sun and Kwok (1987) have also constructed a model of circumstellar $\mathrm{OH}(1612)$ masers. They ignore the chemistry and assume an $\mathrm{OH}$ abundance. The location of the masers is constrained by the assumption of a minimum $\mathrm{OH}$ column density (which is related to the UV optical depth and hence the photoinduced chemistry) required for operating a saturated maser when pumped by $35 \mu \mathrm{m}$ photons. These results give a theoretical explanation of the empirically derived relations between $\dot{M}$ and $R(O H)$ and $L(O H)$.

Even though the $\mathrm{OH}$ masers sample only a short interval of the mass loss history of an individual star, it is still possible to estimate an average time evolution by examining the $\mathrm{OH}$ luminosity function, provided that there is a relation between $\mathrm{L}(\mathrm{OH})$ and $\dot{M}$ (eq.(16)) and that $\mathrm{L}(\mathrm{OH})$ is not very dependent on the MS-mass. Baud and Habing (1983) used this method to derive,

$$
\dot{M}(t)=\dot{M}_{m \text { in }}\left(1-\frac{t}{\tau(\mathrm{OH})}\right)^{-\alpha},
$$

where $\tau(\mathrm{OH})$ is the lifetime of the $\mathrm{OH}$ emitting phase, and $\alpha$ was estimated to be $\approx 0.5$. It was shown by Bedijn (1986) that such a mass loss evolution, with $\alpha \approx 1$, can be expected from the dependence of the scale height of the extended atmosphere on the decreasing stellar mass. In this scenario the Mira variables would correspond to the major portion of $\tau(\mathrm{OH})$, while the $\mathrm{OH} / \mathrm{IR}$-stars appear on the stage shortly before $\tau(\mathrm{OH})$.

\subsection{CO}

Millimetre wave lines of $\mathrm{CO}$ have the advantage, compared to $\mathrm{OH}$, of being detectable towards $\mathrm{O}$-rich as well as $\mathrm{C}$-rich CSEs. The main disadvantage is the limited observational space (see below). In general, the mass loss rate estimated from observations of a $\mathrm{CO}$ line will be an average over a time interval given by eq.(8) or less depending on the excitation requirements. If we assume collisional excitation, i.e., the excitation temperature follows the kinetic temperature, eq.(13), the contribution to the intensity from the region $r$ to $r+d r$ is proportional to $r^{0.3}$ in the optically thick case and $\mathrm{r}^{0.7}$ in the optically thin case (provided that the transition is effectively excited), i.e., more weight is given to the external parts. An estimate of the effectiveness of the excitation is obtained by putting $\exp \left(-E_{u} / k T_{k}(r)\right)=1 / 2$, where $E_{u}=h B J(J+1)$ is the energy of the upper level (B is the rotational constant). The $J \rightarrow J-1$ transition is effectively collisionally excited within, 


$$
r_{1 / 2} \approx 2 \times 10^{17}\left(\frac{2}{J(J+1)}\right)^{1.4} \mathrm{~cm}
$$

This results in $r_{1 / 2} \approx 4 \times 10^{16}$ and $2 \times 10^{15} \mathrm{~cm}$ for the $\mathrm{J}=2-1$ and $6-5$ transitions, respectively. Radiative excitation as well as the inefficiency of collisional excitation at large radii will change this to some extent but the basic behaviour is likely to be correct.

Knapp and Morris (1985) modelled the $\mathrm{CO}(\mathrm{J}=1-0)$ emission from CSEs with spherical geometry, constant mass loss rate, constant expansion velocity, and the kinetic temperature law given in eq.(13). They examined the dependence on the distance, the expansion velocity, the envelope size, and the $\mathrm{CO}$ abundance. For an optically thick line the following simple formula for the mass loss rate can be used,

$$
\dot{M}=\frac{T_{m b} v_{e} e^{2} D^{2}}{A\left(\theta_{b}\right) f(C O)^{0.85}}
$$

where $T_{m b}$ is the main beam brightness temperature of the $\operatorname{CO}(J=1-0)$ line, and $\mathrm{A}\left(\theta_{\mathrm{b}}\right)$ is a constant dependent on the size of the telescope. Eq.(21) is easily applied to data from an arbitrary telescope as long as the CSE is unresolved. For instance, Knapp and Morris derived $A\left(100^{\prime \prime}\right)=1.6 \times 10^{15}\left(\stackrel{M}{\mathrm{M}}\right.$ in $\mathrm{M}_{\theta} \mathrm{yr}^{-1}, \mathrm{v}_{\mathrm{e}}$ in $\mathrm{km} \mathrm{s}^{-1}$, and D in pc), which would scale to $A\left(22^{\prime \prime}\right)=3 \times 10^{16}$ for the IRAM $30 \mathrm{~m}$ telescope. The major sources of error in the derivation of mass loss rates using eq.(21) are, apart from the obvious distance problem (which is more severe here than in the $\mathrm{OH}$ case), the uncertainty in $\mathrm{f}(\mathrm{CO})$, the possible spatial resolution of the CSE, and the use of the same kinetic temperature law for all CSEs.

In the optically thin case the situation becomes more complicated since radiative excitation plays an important role, as opposed to the optically thick case where the excitation is mainly collisional. This is because the infrared transitions become optically thin in the radial direction at the same time as the millimetre wave ones become optically thin in the tangential direction, and this allows infrared photons to flow out into the envelope and take part in the excitation (Schönberg, 1988).

Therefore it is necessary to observationally obtain a $\mathrm{S} / \mathrm{N}$-ratio sufficiently high to be able to define the character of the emission. Nevertheless, van der Veen (1988) has shown that eq.(21) gives roughly the same result as a more sophisticated analysis even in the optically thin case.

We can use eq.(21) to obtain an estimate of the observational space,

$$
\mathrm{D}(\mathrm{CO}) \approx \frac{\mathrm{f}(\mathrm{CO})^{0.4}}{\mathrm{v}_{\mathrm{e}}}\left(\frac{\mathrm{A}\left(\theta_{\mathrm{b}}\right) \dot{M}}{\mathrm{~T}_{\mathrm{mb}}}\right)^{0.5}
$$

For a detection limit of $0.1 \mathrm{~K}$, and assuming $\mathrm{v}_{\mathrm{e}}=15 \mathrm{~km} \mathrm{~s}^{-1}, \mathrm{f}(\mathrm{CO})=5 \times 10^{-4}$, and $\dot{M}=10^{-5} \mathrm{M}_{\theta} \mathrm{yr}^{-1}$, this restricts us in distance to $\mathrm{D}(\mathrm{CO}) \approx 5 \mathrm{kpc}$ even for the IRAM $30 \mathrm{~m}$ telescope. Individual objects more distant than this can of course be detected, in particular when using the $J=2-1$ line (there is a recent detection of an OH/IR-star in the Galactic Centre, Mauersberger et al., 1988), but high mass loss rate objects are rare and for survey work the amount of integration time spent per source is limited.

$\mathrm{CO}$ observations could also be used to sample the mass loss history but it requires considerable observational input (many lines that probe different regions, see eq.(20)) and careful modelling (Sahai, 1987). In some cases the CSEs are large enough to be mapped with sufficient spatial resolution to allow $\dot{M}(t)$ to be derived from the density 
distribution (Huggins et al., 1988; Olofsson et al., 1988a). The sample of sources detected in $\mathrm{CO}$ is far from complete and it is therefore not possible to derive the mass loss evolution from a CO luminosity function. It is also clear from the above discussion that in general at least $\mathrm{CO}(\mathrm{J}=1-0)$ observations result in mass loss rates averaged over a time period given by eq.(8). Comparison of eqs (8) and (18) shows that $t(\mathrm{CO})$ and $t(\mathrm{OH})$ scale almost equally with $\dot{\mathrm{M}}$ and that $\mathrm{t}(\mathrm{OH})$ is only about $10 \%$ of $\mathrm{t}(\mathrm{CO})$. In the case of a mass loss rate changing with time one can therefore expect substantial differences in the mass loss rate estimates based on the two species. If one assumes that the mass loss rate evolves with time according to eq.(19) with $\alpha=1$, and that the $\mathrm{CO}$ and $\mathrm{OH}(1612)$ data samples the mass loss rate at the retarded times given by eqs (8) and (18), respectively, one finds that at the end of the AGB $M(\mathrm{CO}) / \mathrm{M}(\mathrm{OH})=0.2$, and in both cases the mass loss rate is underestimated. It appears that the peak mass loss rate is preferably obtained from IR continuum data. This has been discussed in more detail by van der Veen (1988), who also showed that $\mathrm{OH}$, $\mathrm{CO}$, and IR mass loss rates are in reasonable agreement except in the high mass loss rate range. In fact, for these objects the $\mathrm{CO}$ mass loss rate estimates are unexpectedly low, even taking into account the time evolution, suggesting a decrease in abundance or excitation.

\section{CONCLUSIONS}

It appears now that molecular radio lines can be used to study various aspects of the entire evolution from the early AGB to the PN phase. It is also evident that we have reached such a state of observational maturity that it will be extremely rewarding to analyze the data in considerably more detail with more sophisticated methods.

Acknowledgement. I am grateful to the Swedish Natural Science Research Council (NFR), Kungl. och Hvitfeldtska Stipendieinrättningen, and Wilhelm och Martina Lundgrens Vetenskapsfond for financial support.

\section{References}

Alcock, C., Ross, R.R. (1986a). Ap. J., 305, 837.

Alcock, C., Ross, R.R. (1986b). Ap. J., 310, 838.

Altschuler, D.R., Schneider, S.E., Giovanardi, C., Silverglate, P.R. (1986). Ap. J. (Letters), 305, L85.

Andersson, B.-G., Wannier, P.G., Olofsson, H. (1987). B.A.A.S., 19, 645.

Aspin, C., McLean, I.S., Smith, M.G. (1988). Astr. Astroph., 196, 227.

Bachiller, R., Gomez-Gonzalez, J., Bujarrabal, V., Martin-Pintado, J. (1988). Astr. Astroph., 196, L5

Balick, B. (1987). Astron. J., 94, 671.

Baud, B., Habing, H.J. (1983). Astr. Astroph., 127, 73.

Bedijn, P.J. (1986). In Light on Dark Matter, ed. F. Israel, p. 119, Dordrecht: Reidel. Bedijn, P.J. (1987). Astr. Astroph., 186, 136.

Benson, P.J., Little-Marenin, 1.R. (1987). Ap. J. (Letters), 316, L37.

Bieging, J.H., Chapman, B., Welch, W.J. (1984). Ap. J., 285, 656.

Bieging, J.H., Nguyen-Q-Rieu. (1988a). Ap. J. (Letters), 329, L107.

Bieging, J.H., Nguyen-Q-Rieu. (1988b). preprint.

Bloemhof, E.E., Danich, W.C., Townes, C.H. (1985). Ap. J. (Letters), 299,L37.

Bohlin, R.C., Savage, B.D., Drake, J.F. (1978). Ap. J., 224, 132.

Bowers, P.F., Johnston, K.J., Spencer, J.H. (1983). Ap. J., 274, 733.

Bowers, P.F., Knapp, G.R. (1987). Ap. J., 315, 305. 
Bowers, P.F., Knapp, G.R. (1988). Ap. J., 332, 299.

Bujarrabal, V., Gomez-Gonzalez, J., Bachiller, R., Martin-Pintado, J. (1988). Astr. Astroph., 204, 242.

Cernicharo, J., Guelin, M. (1987). Astr. Astroph., 183, L10.

Chapman, J.M., Cohen, R.J. (1986). M.N.R.A.S., $\underline{220}, 513$.

Cobb, M.L., Fix, J.D. (1987). Ap. J., 315, 325.

Cohen, R.J., Downs, G., Emerson, R., Grimm, M., Gulkis, S., Stevens, G., Tarter, J. (1987). M.N.R.A.S., 225, 491.

Davis, L.E., Seaqvist, E.R., Purton, C.R. (1979). Ap. J., 230, 434.

Deguchi, S., Kawabe, R., Ukita, N., Nakada, Y., Onaka, T., Izumiura, H., Okamura, S. (1988), Ap. J., 325, 795.

Diamond, P.J., Johnston, K.J., Chapman, J.M., Lane, A.P., Bowers, P.F., Spencer, J.H., Booth, R.S. (1987). Astr. Astroph., 174, 95.

Diamond, P.J., Norris, R.P., Rowland, P.R., Booth, R.S., Nyman, L.-A. (1985). M.N.R.A.S., $212,1$.

Dickinson, D.F., Turner, B.E., Jewell, P.R., Benson, P.J. (1986). Astron. J., $22,627$.

Dyck, H.M. (1987). In Late Stages of Stellar Evolution, eds S. Kwok and

S.R. Pottasch, p. 19, Dordrecht: Reidel.

Eder, J., Lewis, B.M., Terzian, Y. (1988). Ap. J. Suppl. Ser., 66, 183

Elitzur, M., Goldreich, P., Scoville, N. (1976). Ap. J., 205, 384.

Fix, J.D. (1987). Astron. J., 92, 433.

Forveille, T., Morris, M., Omont, M., Likkel, L. (1987). Astr. Astroph., 176, L13.

Gathier, R., Pottasch, S.R., Goss, W.M. (1986). Astr. Astroph., 157, 191.

Glassgold, A.E., Huggins, P.J. (1983). M.N.R.A.S., 203, 517.

Goldberg, L. (1986). In The M-type Stars, eds H.R. Johnson and F.R. Querci, p. 245, Washington: NASA SP-492.

Goldreich, P., Scoville, N. (1976). Ap. J., 205, 144.

Habing, H., van der Veen, W., Geballe, T. (1987). In Late Stages of Stellar

Evolution, eds S. Kwok and S.R. Pottasch, p. 91, Dordrecht: Reidel.

Healy, A.P., Huggins, P.J. (1988). Astron. J., 95, 866.

Heiligman, G.M., et al. (1986). Ap. J., 308, 306.

Herman, J., Baud, B., Habing, H.J., Winnberg, A. (1985). Astr. Astroph., 143, 122.

Herman, J., Habing, H.J. (1985a). Phys. Rep., 124, No.4, 255.

Herman, J., Habing, H.J. (1985b). Astr. Astroph. Suppl. Ser., 59, 523.

Hinkle, K.H., Hall, D.N.B., Ridgway, S.T. (1982). Ap. J., 252, 697.

Hodapp, K.-W., Sellgren, K., Nagata, T. (1988). Ap. J. (Letters), 326, L61.

Huggins, P.J. (1987). Ap. J., 313, 400.

Huggins, P.J., Glassgold, A.E. (1982). Astron. J., 87, 1828.

Huggins, P.J., Healy, A.P. (1986a), M.N.R.A.S., 220 , 33P.

Huggins, P.J., Healy, A.P. (1986b), Ap. J. (Letters), 305, L29.

Huggins, P.J., Olofsson, H., Johansson, L.E.B. (1988). Ap. J., 332, 1009.

Jewell, P.R., Schenewerk, M.S., Snyder, L.E. (1985). Ap. J., 295, 183.

Jura, M. (1984). Ap. J., 282, 200.

Jura, M. (1986). Ap. J., $\underline{303}, 327$.

Jura, M., Kahane, C., Omont, A. (1988). Astr. Astroph., 201, 80.

Kahane, C., Gomez-Gonzalez, J., Cernicharo, J., Guelin, M. (1988a). Astr. Astroph., $190,167$.

Kahane, C., Maizels, C., Jura, M. (1988b), Ap. J. (Letters), 328, L25.

Kawabe, R., Ishiguro, M., Kasuga, T., Morita, K.-I., Ukita, N., Kobayashi, H.,

Okumura, S., Fomalont, E., Kaifu, N. (1987). Ap. J., 314, 322.

Keady, J.J., Hall, D.N.B., Ridgway, S.T. (1988). Ap. J., 326, 832.

Knapp, G.R. (1985). In Mass Loss from Red Giants, eds M. Morris and B. Zuckerman, p. 177, Dordrecht: Reidel.

Knapp, G.R. (1986). Ap. J., 311, 731. 
Knapp, G.R. (1987). In Late Stages of Stellar Evolution, eds S. Kwok and S.R. Pottasch, p. 103, Dordrecht: Reidel.

Knapp, G.R., Chang, K.M. (1985). Ap. J., 293, 281.

Knapp, G.R., Morris, M. (1985). Ap. J., 292, 640.

Kwan, J., Linke, R.A. (1982). Ap. J., 254, 587.

Kwok, S. (1975). Ap. J., 198, 583.

Lane, A.P., Johnston, K.J., Bowers, P.F., Spencer, S.H., Diamond, P.J. (1987). Ap. J., $323,756$.

Leahy, D.A., Kwok, S., Arquilla, R.A: (1987). Ap. J., 320, 825.

Lepine, J.R.D., Nguyen-Q-Rieu. (1974). Astr. Astroph., 36, 469.

Lewis, B.M., Eder, J., Terzian, Y. (1985). Nature, 313, 200.

Lewis, B.M., Terzian, Y., Eder, J. (1986). Ap. J. (Letters), 302, L23.

Likkel, L., Forveille, T., Omont, A., Morris, M. (1988). Astr. Astroph., 198, L1.

Likkel, L., Morris, M. (1988). Ap. J., 329, 914.

Likkel, L., Omont, A., Morris, M., Forveille, T. (1987). Astr. Astroph., 173, L11.

Lindqvist, M., Nyman, L. $-\AA$., Olofsson, H., Winnberg, A. (1988). Astr. Astroph., $205, \mathrm{~L} 15$.

Lindqvist, M., Winnberg, A., Matthews, H.E., Habing, H.J., Olnon, F.M. (1987). In Late Stages of Stellar Evolution, eds S. Kwok and S.R. Pottasch, p. 79, Dordrecht: Reidel.

Mamon, G.A., Glassgold, A.E., Huggins, P.J. (1988). Ap. J., 328, 797.

Masson, C.R., et al. (1985). Ap. J., 292, 464.

Mauersberger, R., Henkel, C., Wilson, T.L., Olano, C.A. (1988). Astr. Astroph., 206, L34.

Morris, M., Bowers, P.F., Turner, B.E. (1982). Ap. J., 259, 625.

Morris, M., Guilloteau, S., Lucas, R., Omont, A. (1987). Ap. J., $321,888$.

Morris, M., Jura, M. (1983). Ap. J., 264, 546.

Muchmore, D.L., Nuth, J.A., Stencel, R.E. (1987). Ap. J. (Letters), 315, L141.

Nakada, Y., Deguchi, S., Forster, J.R. (1988). Astr. Astroph., 193, L13.

Nakada, Y., Izumiura, H., Oraka,m T., Hashimoto, O., Ukita, N., Deguchi, S., Tanabe, T. (1987). Ap. J. (Letters), 323, L77.

Nejad, L.A.M., Millar, T.J. (1988). M.N.R.A.S., $230,79$.

Nercessian, E., Guilloteau, S., Omont, A., Benayoun, J.J. (1988). Astr. Astroph., in press.

Netzer, N., Knapp, G.R. (1987). Ap. J., 323, 734.

Ney, E.P., Merrill, K.M., Becklin, E.E., Neugebauer, G., Wynn-Williams, C.G. (1975). Ap. J. (Letters), 198, L129.

Nguyen-Q-Rieu, Epchtein, N., Truong-Bach, Cohen, M. (1987). Astr. Astroph., $180,117$.

Nguyen-Q-Rieu, Laury-Micolaut, C., Winnberg, A., Schultz, G.V. (1979). Astr. Astroph., 75, 351.

Nguyen-Q-Rieu, Winnberg, A., Bujarrabal, V. (1986). Astr. Astroph., 165, 204.

Nyman, L. $\AA$., Olofsson, H. (1986). Astr. Astroph., 158, 67.

Olofsson, H. (1988). Space Science Reviews, 47, 145.

Olofsson, H. Eriksson, K., Gustafsson, B. (1987). Astr. Astroph., 183, L13

Olofsson, H. Eriksson, K., Gustafsson, B. (1988a). Astr. Astroph., 196, L1

Olofsson, H. Eriksson, K., Gustafsson, B. (1988b). in prep.

Olofsson, H. Eriksson, K., Gustafsson, B. (1988c). in prep.

Omont, A. (1987). In IAU Symp. No. 120 Astrochemistry, eds M.S. Vardya and S.P. Tarafdar, p. 357, Dordrecht: Reidel.

Papoular, R., Pegourie, B. (1986). Astr. Astroph., 156, 199.

Payne, H.E., Phillips, J.A., Terzian, Y. (1988). Ap. J., 326, 368.

Pottasch, S.R., Bignelli, C., Zijlstra, A. (1987). Astr. Astroph., 177, LA9.

Reipurth, B. (1987). Nature, $\underline{325}, 787$ 
Ridgway, S.T., Joyce, R.R., Connors, D., Pipher, J.L., Dainty, C. (1986). Ap. J., $\underline{302}, 662$.

Ridgway, S.T., Keady, J.J. (1988). Ap. J., 326, 843.

Rouan, D., Omont, A., Lacombe, F., Forveille, T. (1988). Astr. Astroph., 189, L3.

Rodriguez, L.F., Garcia-Barreto, J.A., Canto, J., Moreno, M.A., Torres-Peimbert, S., Costero, R., Serrano, A., Moran, J.M., Garay, G. (1985). M.N.R.A.S., $215,353$.

Sahai, R. (1987). Ap. J., $318,809$.

Sahai, R., Wannier, P.G. (1985). Ap. J., 299, 424.

Sahai, R., Wannier, P.G. (1988). Astr. Astroph., 201, L9

Schmidt, G.D., Cohen, M. (1981). Ap. J., 246, 444.

Schneider, S.E., Silverglate, P.R., Altschuler, D.R., Giovanardi, C. (1987). Ap. J., 314,572 .

Schönberg, K. (1988). Astr. Astroph., 195, 198.

Sivagnanam, P., LeSqueren, A.M. (1986). Astr. Astroph., 168, 374.

Sopka, R.J., Hildebrand, R., Jaffe, D.T., Gatley, I., Roellig, T., Wemer, M., Jura, M., Zuckerman, B. (1985). Ap. J., 294, 242.

Sopka, R.J., Olofsson, H., Johansson, L.E.B., Nguyen-Q-Rieu, Zuckerman, B. (1988). Astr. Astroph., in press.

Stencel, R.E., Carpenter, K.G., Hagen, W. (1986). Ap. J., 308, 859.

Sun, J., Kwok, S. (1987). Astr. Astroph., 185, 258.

Taylor, A.R., Pottasch, S.R. (1987). Astr. Astroph., 176, L5.

teLintel Hekkert, P. (1987). In Late Stages of Stellar Evolution, eds S. Kwok and S.R. Pottasch, p. 83, Dordrecht: Reidel.

Tielens, A.G.G.M. (1983). Ap. J., 271, 702.

Tsuji, T., Unno, W., Kaifu, N., Izumiura, H., Ukita, N., Cho, S., Koyama, K. (1988). Ap. J. (Letters), 327, L23.

van der Veen, W.E.C.J. (1988). Thesis, University of Leiden.

van der Veen, W.E.C.J., Habing, H.J., Geballe, T.R. (1988). in prep.

Welty, A.D., Fix, J.D., Mutel, R.L. (1987). Ap. J., 318, 852.

Wannier, P.G., Sahai, R. (1986). Ap. J., 311, 335.

Wannier, P.G., Sahai, R. (1987). Ap. J., $319,367$.

Wood, P.R., Bessell, M.S., Whiteoak, J.B. (1986). Ap. J. (Letters), 306, L81.

Zijlstra, A.A., Pottasch, S.R. (1988), Astr. Astroph., 196, L9.

Zuckerman, B., Aller, L.H. (1986). Ap. J., 301, 772.

Zuckerman, B., Dyck, H.M. (1986a). Ap. J., 304, 394.

Zuckerman, B., Dyck, H.M. (1986b). Ap. J., 311, 345.

Zuckerman, B., Dyck, H.M. (1988). Astr. Astroph., in press

Zuckerman, B., Dyck, H.M., Claussen, M.J. (1986). Ap. J., 304, 401. 